\title{
Effect of Cloud Coverage on Sunshine, Humidity, Rainfall and Temperature for Different Weather Stations in Bangladesh: A Panel Analysis.
}

\author{
Md Rokonuzzaman* and Mohammad Maksudur Rahman* \\ "Department of Statistics, University of Chittagong, Chittagong-4331, Bangladesh
}

\begin{abstract}
The aim of the study is to find the effect of the weather variable clould coverage on shunshine, rainfall, humidity and temparature for the different weather sations in Bangladesh. The monthly and yearly data for 33 years (1981-2013) on climatic factors of 13 weather stations in Bangladesh were collected from the Bangladesh Meteorological Department. Data on cloud coverage, total rainfall, temperature, bright sunshine, and relative humidity were collected. It is observed that maximum cloud coverage occurs in the month of July for all the weather stations and it is maximun at the sylhet weather station for every month. Amount of monthly maximum cloud coverage is 7.1 oktas in the month of July and it is minimum with 0.74 oktas at the Cox's Bazar weather station in the month of January. Cloud coverages statistically differ from month to month and station to stations. Significant LM test statistic implies to use Panel model for analyzing the data and insingnificantHausman test statistic support to analyze the panel data with Random Effect Model. The estimated parameters for Random Effect Model found that the weather variable with yearly data humidity and rainfall has positive impact on cloud coverage which is also significant but sunshine and temperature has negative impact where temperature is insignificant. For better prediction one can consider 50 years data to get more accurate result and also can try to perform multivariate analysis such that principle component analysis (PCA), factor analysis, cluster analysis. SARIMA model and Canonical correlation analysis.
\end{abstract}

Key Words: Cloud coverage, shunshine, rainfall, humidity, temparature, Modified regression model,

\section{Introduction}

Clouds are made of tiny drops of water or ice crystals that settle on dust particles in the atmosphere. A cloud is made up of liquid water droplets and it forms when air is heated by the sun. Cloud cover also known as cloudiness, cloudage or cloud amount, refers to the fraction of the sky obscured by clouds when observed from a particular location. Okta is the usual unit of measurement of the cloud cover. The cloud cover is correlated to the sunshine duration as the least cloudy locales are the sunniest ones while the cloudiest areas are the least sunny places.Cloud cover plays an important role in the energetic balance of the atmosphere and a variation of it, is a consequence of and to the climate changeexpected by recent studies. Clouds play multiple critical roles in the climate system.

During the day, the earth is heated by the sun. If skies are clear, more heat reaches the earth's surface. This leads to warmer temperatures.However, if skies are cloudy, some of the sun's rays are reflected off the cloud droplets back into space. Therefore, less of the sun's energy is able to reach the earth's surface, which causes the earth to heat up more slowly. This leads to cooler temperatures. At night cloud cover has the opposite effect. If skies are clear, heat emitted from the earth's surface freely escapes into space, resulting in colder temperatures. However, if clouds are present, some of the heat emitted from the earth's surface is trapped by the clouds and reemitted back towards the earth. As a result, temperatures decrease more slowly than if the skies were clear.

Clouds are important in the earth's climate system because of their effects on solar radiation, terrestrial radiation, and precipitation. These effects depend on cloud height, thickness, horizontal extent, horizontal variability, water content, phase (liquid or ice), and the sizes of droplets and crystals. It is therefore useful to distinguish different types of clouds. The visual appearance of clouds is often taken to indicate characteristic atmospheric dynamical processes, so the traditional classification by weather observers of cloud types based on their appearance continues to be a useful classification in studies of cloud processes.

Cloud amount (or degree of cloud cover) describes the extent of cover by clouds in the portion of the sky visible from the observation point. It is estimated by the weather observer and given in eigth by the synoptic service. The following classification is usually made.

Clear-0-1/10th covered ;Scattered - 1/10th-5/10th covered; Broken - 5/10th-9/10th covered; Overcast - fully covered.In general, clouds tend to form in one of three ways: Firstly,Clouds may form when the air mass is cooled below its dew point. This may happen when the air rises or comes into contact with a cold surface. This can happen where cold and warm air masses collide, and the warmer air (with less density) is lifted 
to greater altitude. It can also happen where air masses are flowing across a mountain range and are lifted for orographic reasons, or it may be caused by convection due to warming of the terrain surface below. Secondly, Clouds may form when warm air blows over a colder surface such as a surface of water with low temperature. and Last way, Clouds can be formed at places where the air temperature increases slowly or remains unchanged, but the air mass rapidly absorbs water vapor from a surface below until it reaches the dew point. This often happens where cold and dry air masses flow across a warm ocean surface without ice cover.

Clouds may also be classified high, middle or low clouds, according to their altitude (the cloud base) above the planet surface. Low clouds primarily reflect incoming solar radiation, whereas high clouds tend to transmit it to the surface but then trap outgoing infrared radiation, contributing to the greenhouseeffect. In Bangladesh cloud remains mostly in the month of June,July,August,September and october. Rest of the months cloud remains comparetively less in the sky.Cloud coverage has a very little affect on humidity. In fact, humidity is mostly affected by the amount of moisture in the air and the amount of moisture found in the air is mostly affected by the amount of water evaporates and rises up in to the atmosphere. The amount of water evaporates is dependent on how warm water in the oceans, lakes, rivers and streams are heated by sunlight and other factors.

Sunshine duration or sunshine hours is a climatological indicator, measuring duration of sunshine in given period usually for a day in a location on Earth. It is a general indicator of cloudiness of a location.Sunshine duration is usually expressed in hours per year, or in hours per day.An important use of sunshine duration data is to characterize the climate of sites, it takes into account the psychological effect of strong solar light on human well-being.

The effects of cloud cover on temperature is a familiar experience. Without a cloud cover in an area the temperature drops sharply at night whereas with clouds the temperature drop is noticeably more moderate. Rain is liquid water in the form of droplets that have condensed from atmosphericwater vapor and then precipitatedthat is, become heavy enough to fall under gravity. Rain is a major component of the water cycle and is responsible for depositing most of the fresh water on the Earth. It provides suitable conditions for many types of ecosystems, as well as water for hydroelectric power plants and crop irrigation.

In order to predict the climate several decades into the future, it is needed to understand many aspects of the climate system, and with the above discussion the following objectives are considered in this study;

- To find the behaviour of the weather variables.

- To find the relationship between cloud cover and some others weather variables.

- To fit a suitable panel model for the cloud cover.

In the next section we will discuss about literature review to this study.

\section{Literature Review}

Reviewing the existing literature on cloud coverage, only a few studies regarding home and abroad.Most of the studies have been conducted to examine the patterns and trend of rainfall based on daily, monthly, seasonal and yearly rainfall data. Only the studies that have dealt with the patterns of cloud coverage are reviewed briefly.Pavelet. al. (1999)examine the Relationship of Cloud Cover to Near-Surface Temperature and Humidity: Comparison of GCM Simulations with Empirical Data and found differences between long-term mean values of surface air temperature and atmospheric humidity for average and clear sky or for average and overcast conditions characterize the long-term non-causal associations between these two elements and total cloud cover. Carole J. (1995) examines The Effect of Moonlight on Observation of Cloud Cover at Night, and Application to Cloud Climatology. They found visual observations of cloud cover arehindered at night due to inadequate illumination of the clouds. This usually leads to an underestimation of the average cloud cover at night, especially for the amounts of middle and high clouds, in climatologies based on surface observations. John W.BergmanandMurryL.Salby(1996) have studied the Diurnal Variations of Cloud Cover and Their Relationship to Climatological Conditions to showthree-dimensional structure of cloud diurnal variations and to investigate their relationship to local climatologicalconditions. YinghuiLiu(2016) have studied the Assessment of Arctic Cloud Cover Anomalies in Atmospheric Reanalysis Products Using Satellite Data. The study examines the performance of five atmospheric reanalysis products and all reanalysis products exhibit biases in the mean cloud amount, especially in winter.Qing Yue et al (2016) studied the Observation-Based Longwave Cloud Radiative Kernels Derived from the A-Train. The authors present a new method to derive both the broadband and spectral longwave observation-based cloud radiative kernels (CRKs) using cloud radiative forcing (CRF) and cloud fraction (CF) for different cloud types using multisensor A-Train observations and MERRA data collocated on the pixel scale. Both observation-based CRKs and model-based CRKs derived from the FuLiouradiative transfer model are shown. Marysa et al (2016)has explained theProgressive Mid-latitude Afforestation: Impacts on Clouds, Global Energy Transport, and Precipitation. They show that the magnitude of afforestation of the northern mid-latitudes determines the local climate response in a non-linear fashion, and we identify a threshold in vegetation-induced cloud feedbacks - a concept not previously addressed by large-scale 
vegetation manipulation experiments. They also show how atmospheric cross-equatorial energy transport changes as the area of afforestation is incrementally increased.

On considering above literature, In this study, statistical analysis on how average clould coverage affected by average of humidity, temparature, humidity, sunshine and total rainfall is done and trying to fit a suitable satatistical panel model of cloud coverage on others climatic variabales.

Data and methods to analysis the data are used in the next section.

\section{Data and Methodology:}

The monthly and yearly data for 33 years (1981- 2013) on climatic factors of several weather stations in Bangladesh were collected from the Bangladesh Meteorological Department, Agargaon, Dhaka, Bangladesh. The collected data were the total rainfall (in $\mathrm{mm}$ ), cloud coverage (in okta), temperature (in ${ }^{0} \mathrm{C}$ ), bright sunshine (in hour's), relative humidity (in \%). For data availability reasons, our investigation focuses on a sample of the following 13 weather stations. The name of the stations are Barisal, Bhola, Bogra ,Chandpur, Comilla, Cox's Bazar, Dhaka, Jessor, Mymensingh, Rajshahi, Rangpur, Sitakunda and Sylhet. Monthly average cloud coverageconsided as dependent variable and all other variables are considered as independent variables. Panel data analysis is done to get more informative data, more variability, less collinearity among variables.Lagranrangemultiliers test statistic is used to decide about using of panel analysis rather than OLS regression and Hausman test test statistic is used for deciding the fixed or random effect in panel model. STATA 12.0, SPSS 19.0 and MS Excel are used to analysis the data and to produce different types of graph. In the next section, discussion are made on the anlytical outputs of data

\section{Results and Discussions}

The monthly avearge cloud cover and its standard error for different weather stations in Bangladesh are shown in Table 1. From the table it is observed that, maximum cloud coverage occurs in the month of july for all the weather stations and minimum cloud coverage occurs in the months of either december or january. It is observed for the Barisal, cloud coverage $6.60 \mathrm{okta} /$ day with SE 0.41 is maximum and $1.01 \mathrm{okta} /$ day with SE 0.81 is minimum for the month of December . For Bhola Station, cloud coverage 6.09 okta/day with SE 0.45 is maximum and $1.02 \mathrm{okta} /$ day with SE 0.58 is minimum for the month of December. Maximun cloud coverage is $6.05 \mathrm{okta} /$ day with SE (0.42) and minimun is 1.09 okta/day with SE (0.60) in december for Bogra wether station. For Chandpur Station, cloud coverage $5.96 \mathrm{okta} /$ day with SE 0.59 is maximum and 1.03 okta/day with SE 0.62 is minimum for the month of January. Maximun cloud coverage is $6.67 \mathrm{okta} /$ day with SE 0.42 and minimun was $0.74 \mathrm{okta} /$ day with SE $(0.47)$ in january for the weather station Cox's Bazar. Cloud coverage is $6.12 \mathrm{okta} /$ day with SE 0.46 which is maximum and minimum is $1.03 \mathrm{okta} /$ day with SE 0.49 for the month of december for the weather station Comilla. For Dhaka Station, cloud coverage 6.45 okta/day with SE 0.29 is maximum and $1.19 \mathrm{okta} /$ day with SE 0.73 is minimum for the month of January. For Jessor Station, cloud coverage $6.13 \mathrm{okta} /$ day with SE 0.46 is maximum and $1.01 \mathrm{okta} /$ day with SE 0.57 is minimum for the month of December. For Mymensingh Station, cloud coverage $6.54 \mathrm{okta} /$ day with SE 0.32 is maximum and 1.18 okta/day with SE 0.57 is minimum for the month of December. For Rajshahi Station, cloud coverage 6.12 $\mathrm{okta} /$ day with SE 0.40 is maximum and $1.02 \mathrm{okta} /$ day with SE 0.63 is minimum for the month of December. For Rangpur Station, cloud coverage $6.46 \mathrm{okta} /$ day with SE 0.42 is maximum and $1.13 \mathrm{okta} /$ day with SE 0.69 is minimum for the month of December. For Sitakundo Station, cloud coverage $6.08 \mathrm{okta} /$ day with SE 0.55 is maximum and $0.98 \mathrm{okta}$ /day with SE 0.69 is minimum for the month of January. For Sylhet Station, cloud coverage $7.10 \mathrm{okta} /$ day with SE 0.25 is maximum and $1.46 \mathrm{okta} /$ day with SE 0.62 is minimum for the month of December.

In the Table1, cloud coverage occurs maximun at the sylhet weather station for every month but minimum cloud coverage does not occur in the same weather station.For the month January, cloud coverage $1.70 \mathrm{okta} /$ day with SE 1.07 is maximum in sylhet station and minimum is $0.74 \mathrm{okta} /$ day with SE 0.42 in Cox's Bazar station.

Table 1Statition wise monthly average cloud coverage(in oktas)with its standard error

\begin{tabular}{|r|l|c|c|c|c|c|}
\hline \multicolumn{1}{|l|}{ SL } & MONTH & Barisal & Bhola & Bogra & Chandpur & Comilla \\
\hline 1 & JANUARY & $1.08(0.61)$ & $1.15(0.59)$ & $1.25(0.56)$ & $1.03(0.62)$ & $1.04(0.65)$ \\
\hline 2 & FEBRUARY & $1.51(0.69)$ & $1.48(0.57)$ & $1.25(0.47)$ & $1.33(0.59)$ & $1.47(0.57)$ \\
\hline 3 & MARCH & $2.50(0.71)$ & $2.17(0.67)$ & $1.59(0.59)$ & $2.15(0.82)$ & $2.31(0.68)$ \\
\hline 4 & APRIL & $3.94(0.65)$ & $3.20(0.47)$ & $3.00(0.72)$ & $3.30(0.77)$ & $3.51((0.53)$ \\
\hline 5 & MAY & $4.93(0.86)$ & $4.14(0.79)$ & $4.25(0.78)$ & $4.26(0.94)$ & $4.54(0.78)$ \\
\hline 6 & JUNE & $6.17(0.47)$ & $5.63(0.54)$ & $5.60(0.55)$ & $5.48(0.78)$ & $5.77(0.46)$ \\
\hline 7 & JULY & $6.60(0.41)$ & $6.09(0.45)$ & $6.05(0.42)$ & $5.96(0.59)$ & $6.12(0.46)$ \\
\hline
\end{tabular}


Effect of Cloud Coverage on Sunshine, Humidity, Rainfall and $\boldsymbol{T}$ emperature for $\boldsymbol{D}$ ifferent ...

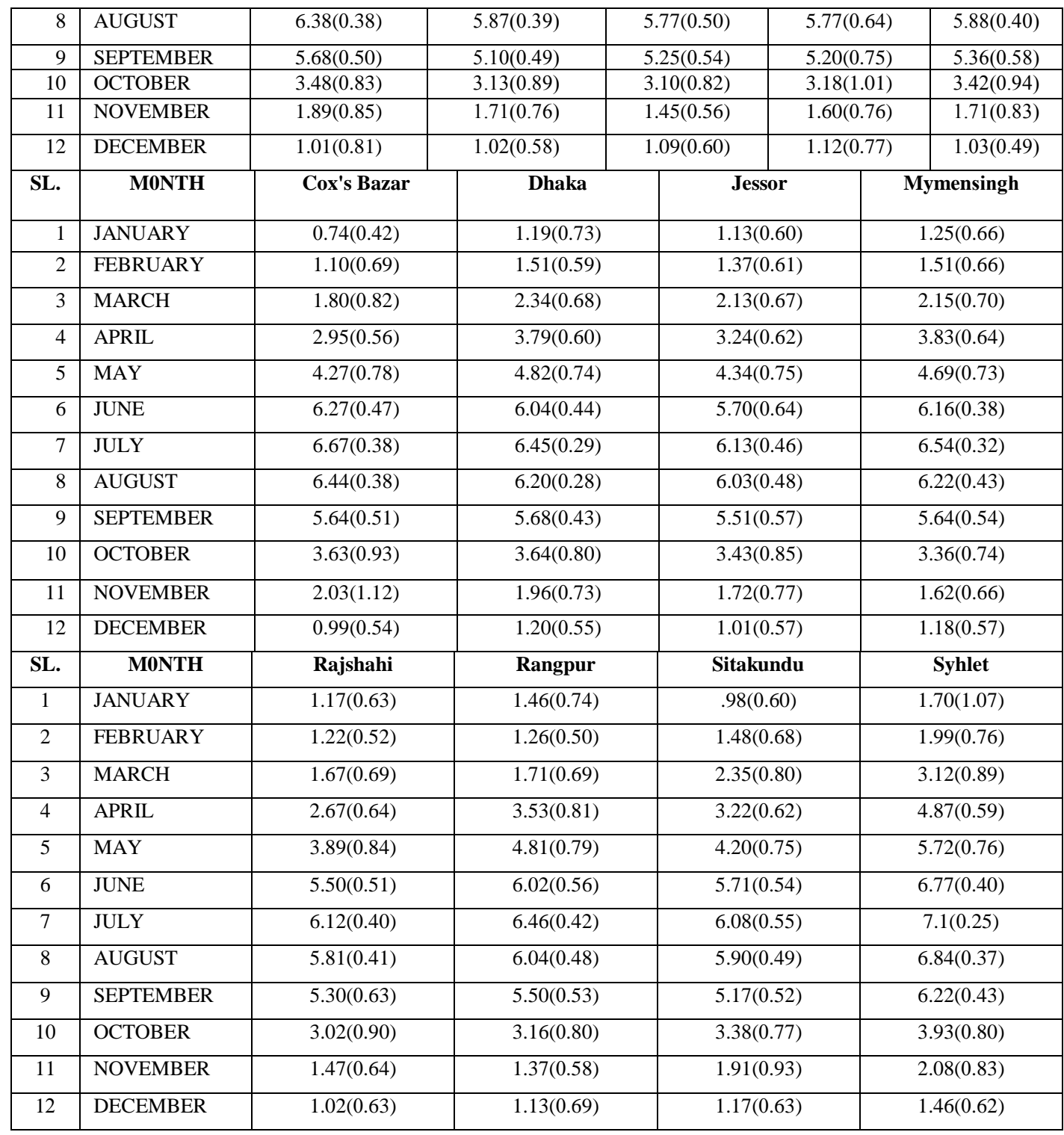

Figures in parenthesis indicates SE

For the month February, cloud coverage 1.99 okta/day with SE 0.76 is maximum in sylhet station and minimum is 1.10 okta/day with SE 0.69 in Cox's Bazar station. For the month March, cloud coverage 3.12 okta/day with SE 0.89 is maximum in sylhet station and minimum is 1.59 okta/day with SE 0.59 in Bogra station. For the month April, cloud coverage $4.87 \mathrm{okta} /$ day with SE 0.59 is maximum in sylhet station and minimum is $2.67 \mathrm{okta} /$ day with SE 0.64 in Rajshahi station. For the month May, cloud coverage $5.72 \mathrm{okta} /$ day with SE 0.76 is maximum in sylhet station and minimum is $3.89 \mathrm{okta} /$ day with SE 0.84 in Rajshahi station. For the month June, cloud coverage $6.77 \mathrm{okta} /$ day with SE 0.40 is maximum in sylhet station and minimum is 5.48 okta/day with SE 0.78 in Chandpur station. For the month July, cloud coverage 7.10 okta/day with SE 0.25 is maximum in sylhet station and minimum is 5.96 okta/day with SE 0.59 in Chandpur station. For the month August, cloud coverage $6.84 \mathrm{okta} /$ day with SE 0.37 is maximum in sylhet station and minimum is $5.77 \mathrm{okta} / \mathrm{day}$ with SE 0.50 in Bogra station. For the month September, cloud coverage $6.22 \mathrm{okta} / \mathrm{day}$ with SE 0.43 is maximum in sylhet station and minimum is $5.10 \mathrm{okta} / \mathrm{day}$ with SE 0.49 in Bhola station. For the month October, cloud coverage $3.93 \mathrm{okta} /$ day with SE 0.80 is maximum in sylhet station and minimum is $3.02 \mathrm{okta} /$ day with SE 0.90 in Rajshahi station. For the month November, cloud coverage 2.08 okta/day with SE 0.83 is maximum in sylhet station and minimum is 1.37 okta/day with SE 0.58 in Rangpur station. For the month of December, cloud coverage $1.46 \mathrm{okta} /$ day with SE 0.62 is maximum in sylhet station and minimum is $0.99 \mathrm{okta} /$ day with SE 0.54 in Cox's Bazar station.Form the Table1, it is obvious that average of cloud coverage (in oktas) per month is maximum (7.1 oktas) at the Sylhet weather station in the month of July and is minimum (0.74) with a low variability $(\mathrm{SE}=0.42)$ at the Cox's Bazar weather station in the month of January. 
Panel analysis:Panel data analysis is used in this study to test the station wise equality of the effect of cloud coverage. Yearwise the average data for cloud coverage, sunshine,humudity and temperature and total rainfall are used as in Yearly time series data, seasonal variability is absent. Fig.1 shows the year wise line diagram of cloud coveragefor different selected weather stations. It is observed from thefigure that for the Sylhet station cloud coverage occurs relatively high compared with other stations.In Chandpur,Jessor,Sitakundo and Cox's Bazar stations there have some fluctuation in cloud coverage whereas the other stations have almost similar patterns. It is observed that more variation is in the Chandpur weather station. Yearly maximum cloud coverage occurs in Sylhet station and minimum is in Chandpur station.

Fig.1 Individual Line diagram for yearly average of cloud coverage for all the selected weather station.

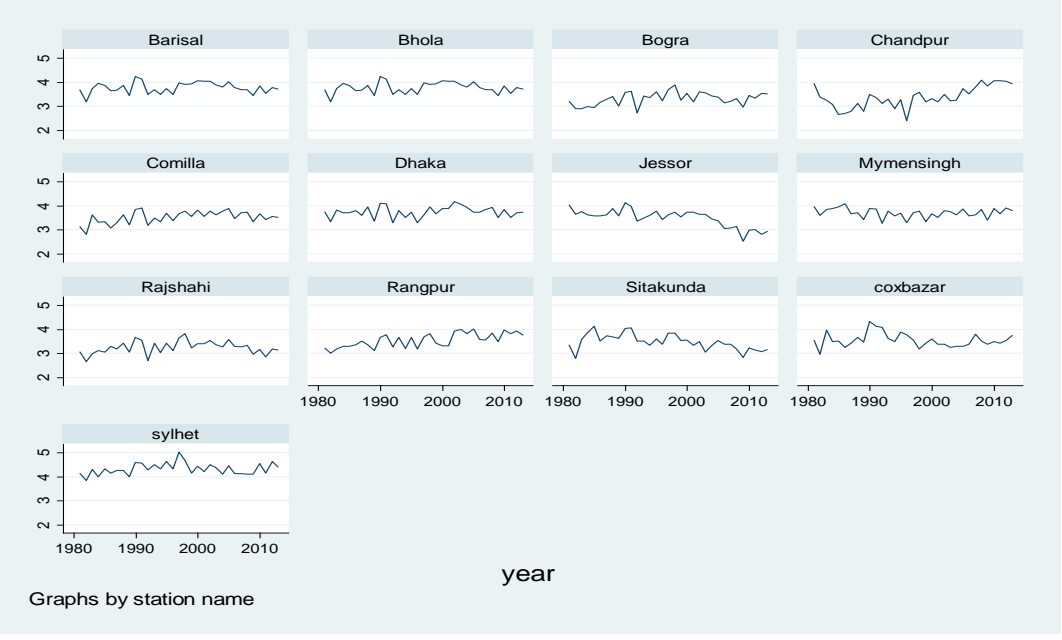

Since it is seemed to have variation among the stations so LM test is used to decide whether the data set is suitable for pooled regression or panel analysis. SignificantLM test statistics (803.21) confered the Panel model is suitable for further analysis. Further by using Hausman test,one can decide the appropriate use of EFM or REM in panel data analysis.For this dataset it is observed that the Hausman test statistic (2.50 with $p$-value 0.645) is insignificant which suggests to use Random Effect Model.

Stata output of estimated parameters for Random Effect Model of cloud coverage on sunshine,humidity,rainfall and temperature is shown in Table 2. In the analysis it is found that the weather variable humidity and rainfall has positive impact on cloud coverage which is also significant but sunshine and temperature has negative impact where temperature is insignificant.

Table 2 Estimated parameters for Random Effect Model (REM) of cloud coverage on sunshine,humidity,rainfall and temperature.

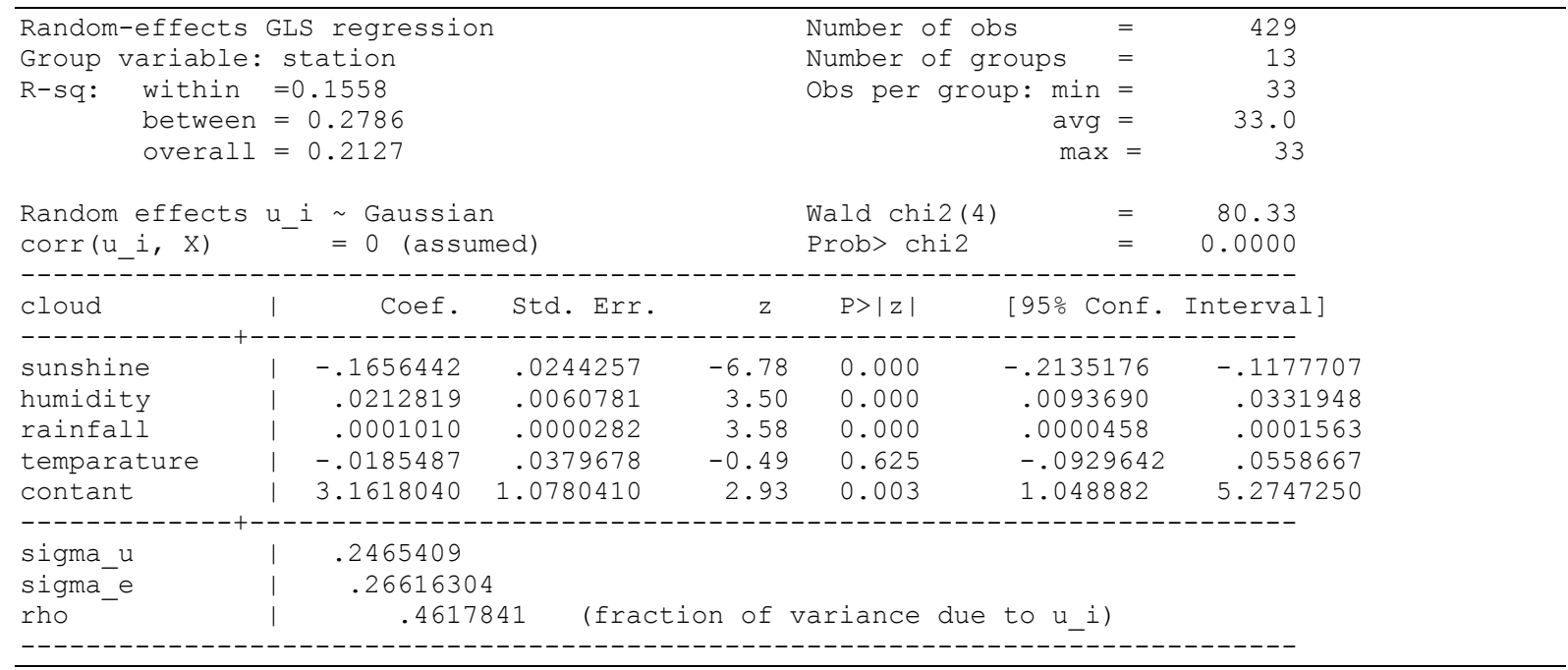




\section{Conclusion}

The aim of the study is to find the effect of clould coverage on shunshine, rainfall, humidity and temparature for the different weather sations in Bangladesh. The summary of the study are following as ;

- Maximum cloud coverage occurs in the month of July for all the weather stations. Cloud coverage occurs maximun at the sylhet weather station for every month.The average cloud coverage per month is maximum (7.1 oktas) at the Sylhet weather station in the month of July.

- The minimum cloud coverage occurs in the months of either December or January for all of these stations. Among the all stations, it is minimum ( 0.74 oktas) with a low variability at the Cox's Bazar weather station in the month of January.

- Line diagram of weather data for year wise data with cloud coverageall the selected weather station have no specific patterns and data seems to be stationary.

- Significant LM test statistic implies to use Panel model for analyzing the data. Further insingnificant Hausman test statistic support to analyze the panel data with Random Effect Model.

- The estimated parameters for Random Effect Model found that the weather variable with yearly data humidity and rainfall has positive impact on cloud coverage which is also significant but sunshine and temperature has negative impact where temperature is insignificant.

\section{References}

[1]. Pavel Y. G., R. S. Bradley and S. Bomin (1999). The relationship of cloud cover to near-surface temperature and humidity: Comparison of GCM simulations with empirical data. Journal of Climate, 13 (11), 1858-1878.

[2]. Carole J. Hahn, Stephen G. Warren and Julius London (1995). The Effect of Moonlight on Observation of Cloud Cover at Night, and Application to Cloud Climatology.Journal of Climate 8(5), 1429-36 . DOI: 10.1175/1520-0442(1995)008.

[3]. John W.BergmanandMurryL.Salby(1996). Diurnal Variations of Cloud Cover and Their Relationship to Climatological Conditions.Journal of Climate 9(11), 2802-20 . DOI: 10.1175/1520-0442(1996)009.

[4]. Yinghui Liu and Jeffrey R. Key (2016). Assessment of Arctic Cloud Cover Anomalies in Atmospheric Reanalysis Products Using Satellite Data. American Meteorological Society, 29, 6065-83. DOI: http://dx.doi.org/10.1175/JCLI-D-15-0861.1

[5]. Qing Yue, Brian H. Kahn, Eric J. Fetzer, Mathias Schreier,Sun Wong

,Xiuhong Chen and Xianglei Huang (2016). Observation-Based Longwave Cloud Radiative Kernels Derived from the A-Train. American Meteorological Society, 29, 5840-49. DOI: http://dx.doi.org/10.1175/JCLI-D-15-0257.1

[6]. Marysa M. Laguë and Abigail L. S. Swann (2016). Progressive Midlatitude Afforestation: Impacts on Clouds, Global Energy Transport, and Precipitation. American Meteorological Society, 29, 5561-72. DOI: http://dx.doi.org/10.1175/JCLI-D-15-0748.1 